\title{
Does Gender Equality Cause Gender Differences in Values? Reassessing the Gender-Equality-Personality Paradox
}

\author{
Filip Fors Connolly ${ }^{1}$. Mikael Goossen ${ }^{1} \cdot$ Mikael Hjerm ${ }^{1}$
}

Published online: 4 December 2019

(C) The Author(s) 2019

\begin{abstract}
The Gender-Equality-Personality Paradox (GEPP) is the finding that gender differences in personality are at their largest in the most gender equal countries. Previous known studies have not examined this relationship over time. Examining this linkage is crucial to our understanding of gender differences and personality development. In the present study, we contrast evolutionary perspectives predicting a gender divergence in personality due to progression in gender equality against biosocial perspectives predicting convergence. Using data from all eight rounds of the European Social Survey $(n=235,339)$ across 32 European countries, we report three findings. First, in accordance with the evolutionary perspective, country-level gender equality is positively associated with gender differences in basic human values. Second, in accordance with the biosocial perspective, we find evidence supporting gender convergence in basic human values. Third, contradicting both evolutionary and biosocial assumptions, we find no evidence that gender equality causes gender differences in values. We argue that there is a need to explore alternative explanations to the observed cross-sectional association between gender equality and personality differences, as well as gender convergence in personality over time.
\end{abstract}

Keywords Gender differences $\cdot$ Personality $\cdot$ Human values $\cdot$ Cross-country comparison $\cdot$ Change over time

An increasing number of studies have investigated differences in personality between men and women across a variety of dimensions such as traits, values, interests, and preferences (Kajonius and Johnson 2018; Zell et al. 2015). This research has shown that gender differences in personality are more palpable in wealthier and more gender-egalitarian countries. Scholars have examined this relationship in a variety of personality-related factors such as basic human values (Schwartz and Rubel-Lifschitz 2009), Big Five personality traits (Mac Giolla and Kajonius 2018), Dark Triad traits (Schmitt et al. 2016), self-esteem (Zuckerman et al. 2016),

Filip Fors Connolly, Mikael Goossen and Mikael Hjerm contributed equally to the present paper.

Electronic supplementary material The online version of this article (https://doi.org/10.1007/s11199-019-01097-x) contains supplementary material, which is available to authorized users.

Filip Fors Connolly

filip.fors@umu.se

1 Department of Sociology, Umeå University, Beteendevetarhuset, Vindarnas torg 1, Umeå, Sweden subjective well-being (Zuckerman et al. 2017), depression (Hopcroft and Bradley 2007), and basic preferences (Falk and Hermle 2018). These results are seemingly paradoxical because one would expect that gender differences in personality decrease rather than increase as opportunities and resources become more evenly distributed, leading to more similar life patterns for men and women. Inspired by Stoet and Geary (2018), we label the positive association between gender equality and gender differences in personality the "genderequality-personality paradox" (henceforth GEPP).

The theoretical complexity surrounding the association between gender equality and gender differences in personality varies. The most common approach is rooted in the tradition of evolutionary psychology (see a review in Schmitt et al. 2017). The evolutionary explanations offered for the GEPP largely build on the assumption of a causal relationship wherein gender equality allows for gender differences in personality. For instance, Schmitt et al. (2017) posit that gender differences in personality are innate, due to sexual selection in our ancestral past, and is manifested in societies to a varying degree depending on their level of gender equality and economic development. Likewise, Falk and Hermle (2018) state that increased availability of resources, as well as 
gender-equal access to resources, enable the independent development and manifestation of gender-specific preferences.

The positive cross-sectional pattern of gender equality and gender differences in personality is robust. However, we argue that the casual language framing these correlational observations may be premature because no known study has tested this relationship over time. In addition, when studying the development of gender differences in personality, some studies point to a convergence rather than a divergence (Donnelly and Twenge 2017; Twenge 1997). These results, rooted in the biosocial perspective on gender differences (Wood and Eagly 2012), are seemingly at odds with the assumption underlying GEPP

Gender differences can certainly be larger in more genderequal countries while also converging over time. However, gender equality cannot simultaneously be the cause of both convergence and divergence. Thus, we set out to examine how changes in gender equality relate to changes in personality (e.g., basic human values in the present case). To be able to ascertain that a contextual-level factor causes an effect, one must be able to demonstrate that variation in the causing factor temporally precedes variation in the outcome. One must also study a large enough sample of countries to demonstrate that this is a generalizable relationship. Only a cross-country longitudinal approach, studying both changes in gender equality and changes in personality within the same model, can remedy the shortcomings of previous approaches within both the evolutionary and the biosocial traditions. We use European Social Survey data between 2002 and 2016 (eight rounds) for 32 countries to test if changes in gender equality drive a process of gender divergence or convergence in personality.

The present article is organized as follows. First, we delineate the two main theoretical approaches to gender differences in personality: the evolutionary and the biosocial perspective. We then turn to the relationship between gender equality and basic human values and present three hypotheses. Next, we outline the data, method and analytical strategy before presenting the results. The article ends with a concluding discussion.

\section{Evolutionary Perspectives on Gender Differences in Personality}

Evolutionary theories view psychological gender differences as partly due to innate dispositions, which are developed in response to different adaptive problems faced by men and women in our ancestral past (see reviews in Schmitt et al. 2008, 2017). Although the mechanism is sexual selection (Bjorklund and Shackelford 1999), manifest gender differences are regarded as a complex interaction between biology and the socioecological context. This interplay results in varying situational and developmental life-stage gender differences in personality (see review in Schmitt et al. 2017). For instance, according to the curvilinear hypothesis of cultural variation, agricultural societies with large inequalities in income distribution and gender inequality deviate more from hunter-gatherer societies than do the conditions of modern societies (Schmitt et al. 2008). Thus, more economically developed societies should display larger sex/gender differences in personality than less developed agrarian societies.

Similarly, post-materialist theory (Falk and Hermle 2018) posits that more economically developed and gender-equal societies should display the largest psychological sex/gender differences because such conditions allows men and women to more freely express their intrinsic dispositions (Falk and Hermle 2018). It is also suggested that environmental pressures, such as malnutrition or differences in subsistence strategies, related to physiological sex dimorphism could suppress the development of inherent sex/gender differences in personality as well because both sex dimorphism and sex/gender differences in personality relate to measures of human development (Schmitt et al. 2017).

Studies utilizing a cross-sectional, comparative research design are relatively consistent in reporting a positive correlation between different indicators of gender equality and gender differences in measures of personality. For instance, indicators of gender equality have been found to correlate with multivariate measures (Mahalanobis' $d$ ) of gender differences in Big Five personality traits, ranging from $r=.44$ (Kaiser 2019) to $r=.69$ (Mac Giolla and Kajonius 2018). Similarly, Schmitt and colleagues (Schmitt et al. 2008, p. 177) found positive correlations between a General Sex Difference Index, measuring gender differences in Big Five traits, and general measures of equality such as a Gender Empowerment Measure $(r=.39)$ and the Gender-Related Development Index $(r=.49)$. They also found correlations with more specific measures such as sex/gender differences in life expectancy $(r=.45)$, proportion of female professionals and workers $(r=.45)$, and sex differences in blood pressure $(r=.31)$.

Looking at basic preferences (for altruism, trust, positive and negative reciprocity, risk-taking, and patience), Falk and Hermle (2018) found average gender differences to be positively correlated with a Gender Equality Index $(r=.56)$ (covering material, social, and political gender equality). Schwartz and Rubel-Lifschitz (2009) found evidence that gender differences are positively correlated with a Gender Equality Index for six of ten basic human values in 25 European countries. Findings also point to positive associations between gender equality and adjacent outcomes such as gendered occupational and educational choices (i.e., number of men/women within STEM [Science, Technology, Engineering, and Math] and EHW [Education, Health and Welfare] fields (Stoet and Geary 2018), self-esteem (Zuckerman et al. 2016), subjective well-being (Zuckerman et al. 2017), and depression (Hopcroft and Bradley 2007). 
Although the empirical evidence of a positive correlation between gender equality and gender differences in personality is overwhelming, we identify some issues with the proposed explanations that remain underdeveloped or represent inconsistencies. For example, we are concerned with arguments suggesting that inherent gender differences could be amplified in societies where socially construed gender norms are relaxed (Mac Giolla and Kajonius 2018). For instance, Falk and Hermle (2018) state that gender equality prompts gender differences due to women's lessened exposure and vulnerability to male influence paired with greater opportunities for selfexpression. Does this mean that, they argued, suppression of inherent gender differences in less gender-equal countries are due to prevailing gender norms socializing men into being more feminine and/or women into being more masculine? Specifically, does it mean that men influence women to adopt masculine rather than feminine preferences? In addition, such explanations are seemingly at odds with evidence suggesting that cross-cultural variation is primarily due to variations in men's personality scores (Schmitt et al. 2008).

Furthermore, whereas it is relatively easy to follow how a lack of material resources could decrease sex differences in physical attributes such as height (e.g., due to malnutrition), it is more difficult to imagine how equality of resources would increase gender differences in personality (cf. Schmitt et al. 2017, p. 49). If, for instance, malnutrition would reduce the development of innate sex differences in the brain, one would primarily expect variation in gender differences in personality when comparing poor countries to rich countries. Yet, the available correlative evidence points to a linear relationship between gender equality and personality differences even within affluent countries (Mac Giolla and Kajonius 2018; Schwartz and Rubel-Lifschitz 2009).

The most explored alternative explanation to the correlative evidence of the GEPP is measurement artifacts related to a variety of different measurement issues. However, Kaiser (2019), for instance, shows that correlative evidence of GEPP holds even after controlling for measurement equivalence. Furthermore, a study by Schmitt et al. (2008), covering 55 cultures, tested for measurement artifacts by relating interitem response variance, acquiescence bias, negative item bias, and Cronbach's alphas to gender differences in personality. The results indicate that gender differences in personality are not due to differences in response bias or measurement error. In addition, studies on educational choice display the same pattern of gender differentiation as self-reported personality measures (Wang and Degol 2017). Taken together, although we cannot rule out this possibility, measurement error as an explanation of the GEPP is not supported by available research.

Another possible explanation is differences between geographical regions, with some evidence suggesting that gender differences are primarily found in Europe and the Americas (which exhibit moderate-to-large differences) versus all other regions (which exhibit small-to-no differences, with some variation) (Schmitt et al. 2008). Furthermore, Kaiser (2019) found that the cross-sectional association between gender equality and gender differences in the Big Five personality facets disappears when controlling for historic pathogen prevalence, food availability, and cultural individualism. Thus, empirical evidence suggests it is difficult to disentangle the effects of gender equality from other aspects of human development, further highlighting the need to develop a coherent theoretical framework specifying the proposed causal connection between gender equality and personality.

\section{Biosocial Perspectives on Gender Differences in Personality}

The biosocial constructionist theory posits that societal gender specialization coincides with gender differences in traits, values, preferences, and behavior (Wood and Eagly 2012). This perspective holds that gender differences in personality are a consequence of internalized gender roles derived from the gender division of labor in society (Eagly et al. 2000; Wood and Eagly 2012). Observations of men and women performing different tasks in society are erroneously attributed to innate differences along lines of the broad grouping of traits into communal (i.e., warm, nurturing, and relation-oriented) and agentic (i.e., instrumental, assertive, and achievement-oriented) domains. Expectations about the behavior of men and women are integrated into personality by way of selfcategorization and self-stereotyping (facilitated by sanctions and approval, neurological reward responses, and the situational elicitation of hormones) (Wood and Eagly 2012). The expectation is that gender equality leads to a convergence of life patterns and experiences, resulting in a convergence of personality between men and women.

Research emanating from the biosocial perspective provides several accounts of trends in gender differences in personality. The overall conclusion is that convergence in personality is more prominent than divergence. For example, gender differences in masculine and feminine traits in the United States show a trend toward convergence. Men's masculinity and femininity scores on the Bem Sex Role Inventory scale remained stable whereas women's androgyny scores increased significantly from 1974 to 1993 (Donnelly and Twenge 2017). The change for women was due to an increase in self-reported masculine traits up to 1993 , followed by a significant decrease in femininity scores between 1993 and 2012. This convergence took place during a period when gender equality increased in the United States (U.S. Census Bureau 2012).

Other aspects of personality show signs of convergence rather than divergence as well. For instance, mate preferences 
(studied 1939-2008) trend toward convergence as women increasingly value attractiveness and decreasingly value financial resources, whereas resources have become more important in men's mate preferences (Boxer et al. 2013). Meanwhile, the correlation between socioeconomic status and self-esteem has decreased over generations for men, but increased for women (Twenge and Campbell 2002). Women's occupational goals and preferences have altered over time to more closely resemble men's (Konrad et al. 2000). Compared to women, the tendency of men to emerge as leaders in initially leaderless groups has diminished over time (Eagly and Karau 1991). Traits, such as narcissism, which are connected to leadership positions, display mixed evidence over time, with both evidence of convergence (Twenge et al. 2008) as well as stability (Grijalva et al. 2015).

The studies we reported here do not necessarily focus on the same aspects or use the same measures of personality as the studies that have reported a cross-sectional gender-equality paradox. However, important similarities certainly exist. For instance, Donnelly and Twenge's (2017) measure of masculinity scores (which display gender-convergence over time in the U.S.) contains items like "dominant," "aggressive," and "competitive" - personality characteristics conceptually related to low scores on the Big Five personality trait, Agreeableness, as well as to high scores on Power and Achievement values. All these factors display larger gender differences in more gender-equal countries (Mac Giolla and Kajonius 2018; Schwartz and Rubel-Lifschitz 2009). However, perhaps the most pressing issue with the biosocial perspective is a lack of studies modeling level of gender equality against gender differences in personality across multiple countries and across time.

\section{The Gender-Equality-Personality Paradox and Values}

Previous studies on GEPP have operationalized personality in a variety of ways with similar results (Schmitt et al. 2017). In the present study, we focus on basic human values as one aspect of personality. Following Schwartz (1992), we define values as broad, trans-situational goals, which vary in importance and that act as guiding principles in people's lives. Because values are critical motivators of behaviors and preferences (Schwartz 2012), values can mediate the relationship between gender and different outcomes (such as educational and occupational choice). Furthermore, values provide a good middle ground between previously studied higher-order traits (e.g., Big Five Personality Traits; Mac Giolla and Kajonius 2018) and lower-order aspects of personality such as basic preferences (Falk and Hermle 2018).

Value priorities, being correlated with higher-order personality traits (Fischer and Boer 2015), also display gender differentiation and cross-cultural variations similar to those found for traits and preferences (Schwartz and RubelLifschitz 2009; Falk and Hermle 2018). Schwartz and Rubel (2005) studied 127 samples from over 70 countries and found that men scored higher than women on the value domains of Power, Stimulation, Hedonism, Achievement, and Self-Direction. Women scored higher than men on Benevolence and Universalism. In a follow-up study (of 25 nationally representative adult samples and student samples representing 68 countries), gender differences in value priorities were found to vary in size across countries according to their level of gender equality (Schwartz and Rubel-Lifschitz 2009). More gender-equal countries displayed the largest differences. Finally, although values were previously thought to be generationally transmitted through socialization, recent empirical advancements suggests that value priorities are due to both genetic and social factors (Uzefovsky et al. 2016). This finding makes both the biosocial and evolutionary perspectives applicable to our analysis.

\section{Hypotheses}

Because recent cross-sectional research adopting an evolutionary approach finds positive correlations between levels of gender equality and size of gender differences in traits and value priorities, we posit that gender equality will be positively correlated with gender differences in value priorities (Hypothesis 1). However, empirical research emanating from the biosocial perspective finds that, over time, personality is converging on several measures, such as the Bem Sex Role Inventory. We thus posit that gender differences within each of five value priorities will converge over time across countries (Hypothesis 2).

Finally, the two main theoretical perspectives predict opposite causal directions. The evolutionary perspective predicts that gender equality drives a process of gender divergence in personality because greater equality of resources and increased autonomy enables each sex to better pursue their innate dispositions, which were acquired through sexual selection in our ancestral past (e.g. Schmitt et al. 2017). The biosocial perspective predicts that progression in gender equality leads to altered gender roles, with life patterns and expectations overlapping to a greater extent, thus driving a process of convergence in values (Wood and Eagly 2012). Drawing upon this reasoning, we posit two competing hypotheses: (a) Progression in gender equality will drive gender divergence in values (i.e., an evolutionary approach) (Hypothesis 3a) or (b) Progression in gender equality will drive gender convergence in values (i.e., a biosocial approach) (Hypothesis 3b). 


\section{Method}

\section{Participants}

We used data from all eight waves of the European Social Survey (ESS 2016). ESS is a cross-sectional biannual survey with representative samples of persons aged 15 and older in Europe. Face-to-face interviews are conducted with a random sample of approximately 1500-2000 respondents in each wave and country. The time-period spanned from 2002 to 2016 and included 32 countries. However, not all included countries participated in all rounds (see Table 1s in our online supplement). All in all, 235,339 respondents participated.

The representation of respondents in relation to nonrespondents are generally good in ESS, but as in most large surveys, there tends to be some underrepresentation of young people, non-nationals, men, and single households to varying degree in different countries (e.g., see Koch 2018, for ESS round 7). We applied stratification weights to ensure better representativeness. In one of our robustness checks we also used data from the International Social Survey Programme. (Results are available in Table $2 \mathrm{~s}$ in our online supplement.)

\section{Measures}

\section{Value Priorities}

The dependent variables were value priorities. We focused on the following five basic human values based on the findings and theoretical discussion in Schwartz and Rubel-Lifschitz (2009): Achievement, Benevolence, Power, Stimulation, and Universalism. Participants completed a revised version of the Portrait Values Questionnaire (PVQ; Schwartz 2003), which was available in majority and large minority languages for all participating countries (following TRAPD translation protocols, Capstan translation verification, and survey quality predictor coding). Two or three items representing verbal portraits of individuals with different goals and aspirations were used to measure each value. Respondents answered by rating how similar they were to each portrait using a 6-point labelled scale ranging from 1 (not like me at all) to 6 (very much like $m e$ ). Table 1 displays the core motivational goal for each value type, its survey items, and the average Cronbach's alpha for the value measures across all countries. The somewhat low reliability for some estimates (e.g., .41 for Power) are expected because the items were intended to capture the conceptual breadth of the value rather than its's core concept (see

Table 1 Description of values used in the present study

\begin{tabular}{|c|c|c|}
\hline Value Type & Core Motivational Goal & Survey Items \\
\hline Achievement $(\alpha=.70)$ & $\begin{array}{l}\text { Personal success through demonstrating } \\
\text { competence according to social standards }\end{array}$ & $\begin{array}{l}\text { It's important to her to show her abilities. She wants people } \\
\text { to admire what she does. } \\
\text { Being very successful is important to her. She hopes people } \\
\text { will recognize her achievements. }\end{array}$ \\
\hline Benevolence $(\alpha=.61)$ & $\begin{array}{l}\text { Preservation and enhancement of the welfare of } \\
\text { people with whom one is in frequent personal } \\
\text { contact }\end{array}$ & $\begin{array}{l}\text { It's very important to her to help the people around her. } \\
\text { She wants to care for their well-being } \\
\text { It is important to her to be loyal to her friends. She wants } \\
\text { to devote herself to people close to her. }\end{array}$ \\
\hline Power $(\alpha=.41)$ & $\begin{array}{l}\text { Social status and prestige, control or dominance } \\
\text { over people and resources }\end{array}$ & $\begin{array}{l}\text { It is important to her to be rich. She wants to have a lot of } \\
\text { money and expensive things. } \\
\text { It is important to her to get respect from others. She wants } \\
\text { people to do what she says. }\end{array}$ \\
\hline Stimulation $(\alpha=.64)$ & Excitement, novelty, and challenge in life & $\begin{array}{l}\text { She likes surprises and is always looking for new things to do. } \\
\text { She thinks it is important to do lots of different things in life. } \\
\text { She looks for adventures and likes to take risks. She wants to } \\
\text { have an exciting life. }\end{array}$ \\
\hline Universalism $(\alpha=.59)$ & $\begin{array}{l}\text { Understanding, appreciation, tolerance and } \\
\text { protection for the welfare of all people and } \\
\text { for nature }\end{array}$ & $\begin{array}{l}\text { She thinks it is important that every person in the world be } \\
\text { treated equally. She believes everyone should have equal } \\
\text { opportunities in life. } \\
\text { It is important to her to listen to people who are different from } \\
\text { her. Even when she disagrees with them, she still wants to } \\
\text { understand them } \\
\text { She strongly believes that people should care for nature. } \\
\text { Looking after the environment is important to her. }\end{array}$ \\
\hline
\end{tabular}

Note. Alphas are averaged across the 32 countries. Survey items are taken from the female version of the PVQ 
Schwartz and Rubel-Lifschitz 2009) and because the number of items for each construct was small (i.e., 2-3).

Previous studies support the reliability and validity of the Portrait Values Questionnaire. Using data from ESS Wave 1, Piurko et al. (2011) separately tested each of the value types we use in our study and obtained metric invariance across 20 countries. Furthermore, studies have shown that the Portrait Values Questionnaire has good predictive validity because the measures correlate in the expected directions with behaviors such as voluntary memberships and political activism (see Schwartz 2007).

We followed Schwartz (2003) and used centered scores to assess the importance of each value to that person. The benefit of this procedure is that it measures value priorities, meaning the importance of each value in relation to all ten original values measured with the PVQ (with the benefit of also accounting for different response styles among respondents). First, we averaged each of the items that belonged to the same value type into an index and then calculated the relative importance of that value by mean centering each respondent on his or her own mean of all ten original value domains. Second, we calculated the average country scores for each value for men and women and for the absolute difference between them. Higher values indicate a larger gender difference, without discriminating between which gender scored higher. A decrease in the dependent variable signifies convergence and an increase signifies divergence.

As a robustness check, we cleaned data to correct for nondiscriminatory response styles (same answer to more than 16 items) or respondents with missing information on five of 21 items. This check did not significantly affect gender differences. Hence, all data were retained in our final analyses.

\section{Gender Equality Index}

The independent variable is Gender Equality Index (Gender Inequality Index reversed). The Gender Equality Index (GEI) was collected by the United Nations Development Programme (UNDP 2018). The index is a composite of health, empowerment, and labor market participation. GEI was only available for 2000 (1995 for the Czech Republic and Slovenia), 2005, and every year from 2010 and onward. We linearly imputed values for the missing years using the adjacent values. GEI changed relatively evenly within countries over years so the imputation procedure is not likely to misrepresent the true scores. All countries increased their gender equality over time, but to varying degrees. On a 0 to 1 scale (higher scores indicating higher gender equality), countries ranged from .43 (Turkey in 2004) and .96 (Denmark, Norway, Sweden, Switzerland in 2016). The GEI grand mean was .84 in 2002 and .92 in 2016. The within-country change in GEI between 2002 and 2016 was on average .06, ranging from .01 in Sweden to .15 in Poland.

\section{Analysis Plan}

In order to assess gender divergence/convergence in value priorities, we applied repeated measurement models where countries were nested in time. Thus, we modelled gender differences longitudinally where we examined if change in gender equality was associated with changes in gender difference in values. To be able to parse out the between country differences from within-country change we mean-centered gender equality for each country and subtracted the raw yearly score from the grand mean. This procedure resulted in two orthogonal variables: one accounting for the between-country differences (time fixed covariate) and the other accounting for within-country change (time varying covariate). The grand mean centering was done on all valid years for each country (i.e., for countries with missing data on the dependent variable, the mean centering was executed on the independent variable matching those observations).

Time was modelled as a continuous variable in all analyses. Other options were tried (like including a quadratic time parameter), but all resulted in poorer model fit. Depending on the model, there were slight differences in the covariance structures. For the empty models where we only included time, we used an unstructured random covariance structure, combined with an identity repeated structure. Due to the lack of covariance between intercept and slope, we simplified the random structure in the subsequent analyses. The models including independent variables used identity covariance combined with an autoregressive repeated part. This accounted for correlation between time points because two adjacent observations are expected to be more correlated than two distant observations.

\section{Results}

\section{Preliminary Analysis}

We start our analysis with descriptive analyses and some tentative tests of Hypothesis 1, which predicted that gender equality would be positively correlated with gender differences in value priorities. First, we replicated the crosssectional association between gender equality and gender differences in personality by correlating GEI with the values. For the individual values, the correlation coefficients (Pearson's $r$ ) are .30 for Achievement $(p=.096), .62$ for Benevolence $(p<.001), .47$ for Power $(p=.007), .47$ for Universalism $(p=.007)$, and .15 for Stimulation $(p=.480)$. Although three of the five bivariate correlations are statistically significant, the direction of all relationships are in the expected direction. To obtain a broader measure of personality differences, we also calculated an index that captures the average gender difference across all five values. The correlation between this index and GEI was $.55(p<.001)$. Together these findings 
Table 2 Standardized gender differences in values in pooled ESSsamples (17 countries)

\begin{tabular}{lllll}
\hline & \multicolumn{2}{l}{ Cohen's $d$} & & \\
\cline { 2 - 4 } Values & $2002-2004$ & $2014-2016$ & Difference & Reduction \\
\hline Achievement & .28 & .23 & .05 & $19.4 \%$ \\
Benevolence & .35 & .32 & .03 & $9.2 \%$ \\
Power & .30 & .25 & .06 & $19.5 \%$ \\
Stimulation & .21 & .19 & .02 & $11.8 \%$ \\
Universalism & .27 & .23 & .04 & $15.1 \%$ \\
Average & .28 & .24 & .04 & $15.0 \%$ \\
\hline
\end{tabular}

mimic previous cross-sectional studies. For example, Falk and Hermle (2018) report a correlation of .56 between gender equality and a summary index of gender differences in preferences. Our tentative analysis renders initial partial support for Hypothesis 1.

Turning to Hypothesis 2, which hypothesized that gender differences in all five value priorities would converge over time across countries, Fig. 1 shows the average crosscountry difference in values between men and women between 2002/2004 to 2014/2016 for the 17 available countries. We average the differences for the last two rounds and subtract the average from the first two rounds of ESS to minimize yearto-year fluctuations. Negative scores indicate a convergence in values between men and women and positive values indicate a divergence. The pattern is fairly consistent across countries in the tendency toward more convergence on all five values. Switzerland is deviating from the pattern because all values hint at a slight tendency toward divergence. There are also deviations from the convergence pattern on various values in different countries even though the main pattern leans toward convergence.

A crude way of illustrating this pattern is by pooling all samples (countries) and comparing the standardized difference (Cohen's $d$ ) between men and women in the first two waves of ESS (2002-2004) to the last two waves of ESS (2014-2016). Table 2 shows that Cohen's $d$ decreases for all values with an average reduction of $15 \%$. This very simple description of the data is in accordance with Hypothesis 2, which states that men's and women's value priorities converge over time.

\section{Longitudinal Models}

Turning to the longitudinal models, we first ran repeated measurement models with only time (see Table 3 ). Negative time coefficients mean that the difference between men and women is decreasing over time (i.e., converging) because the coefficients correspond to the average change in value differences between men and women across time points. Looking at specific values, Benevolence, Power, and Achievement are converging over time, whereas Universalism and Stimulation display no significant change over time. The effects are not substantial but they clearly refute the idea of a divergence in these values because men and women are becoming more alike over time. Hypothesis 2, predicting a gender convergence in values, is thus partly confirmed. Moreover, there is significant variation in intercept across countries ( $b=.15-.23, p<.001)$, but little or no variation in slope (Power: $p=.040$, all other $p \mathrm{~s}>.22$. In other words, the rate of change in value differences does not vary across countries. There is generally no covariance between intercept and slope, which indicates that the change in gender differences in values is not related to the size of the gender difference.

Table 4 includes the main predictor of gender differences: GEI (Gender Equality Index). The table shows that there are cross-country differences (GEI-between) in values significantly related to Benevolence ( $b=.39, p<.001)$, Universalism $(b=.26, p=.001)$, and Power $(b=.45, p=.002)$. The effects are the average difference between men and women in value priorities related to a one-unit difference in GEI between countries (i.e., the more gender equality in a country, the larger the difference in values between men and women). This is in accordance with Hypothesis 1, which is confirmed.

In order to test our competing hypotheses-Hypothesis 3a, which posited that a progression in gender equality will drive gender divergence in values (i.e., a positive effect), and Hypothesis 3b, which proposed that a progression in gender equality will drive gender convergence in values (i.e., a negative effect) - we focus on within-country change (i.e., GEI within). This measure indicates a significant negative effect for GEI on Stimulation $(b=-1.16, p=.013)$ but nonsignificant effects for the other four values (all $p \mathrm{~s}>.40$. The effects are the average changes in gender differences in values related to a one-unit increase in GEI. This pattern finds no support for Hypothesis $3 a$ and scant support (i.e., only for Stimulation) for Hypothesis $3 \mathrm{~b}$. Overall, the general results do not support the idea that changes in gender equality drive gender differences in values.

However, before finally refuting Hypothesis 3 , we need to account for the possibility that the changes in values are more responsive to long-term effects. Therefore, we also test a number of growth models where we account for the possibility that differences in gender values are driven by onset levels of gender equality rather than short-term feedback effects. We examine if gender equality at the first time point drives changes in value differences. It is important to note that countries with high levels of gender equality in 2002 also tend to be countries that had higher levels of gender equality further back in time (Paxton et al. 2008).

Table 5 shows that there is no significant interaction effect between time and gender equality (all $p \mathrm{~s}>.08$. This means that the level of GEI in 2002 does not relate to changes in 
Benevolence

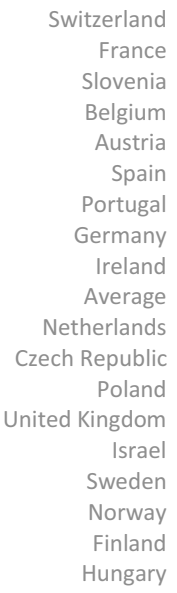

$-0.3$

$-0.1$

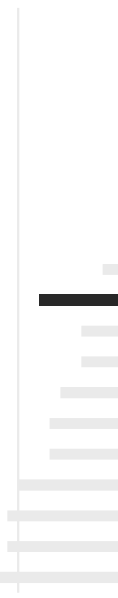

Power

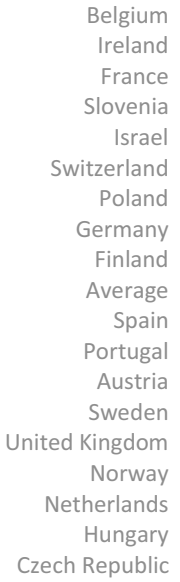

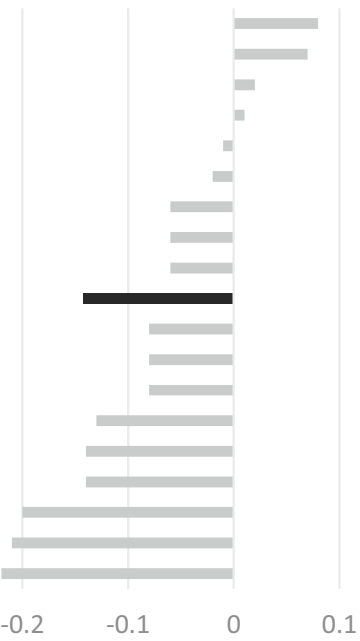

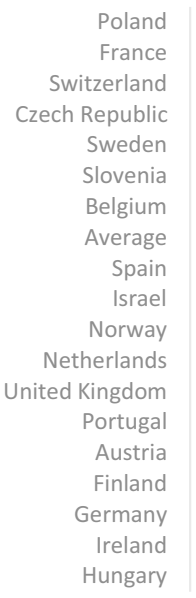

$-0.3$

Achievement

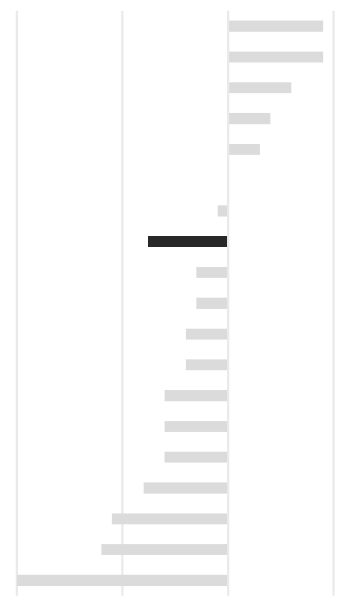

Switzerland
Belgium
Czech Republic
Spain
Israel
France
Slovenia
Portugal
Poland
Average
Ireland
Netherlands
Austria
United Kingdom
Norway
Finland
Hungary
Sweden
Germany

$-0.3$

Universalism

$-0.2$

0.1

0.1

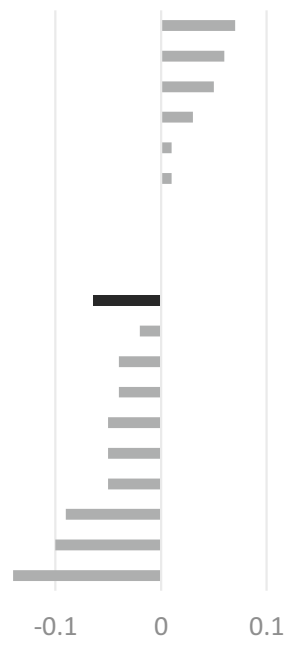

Stimulation

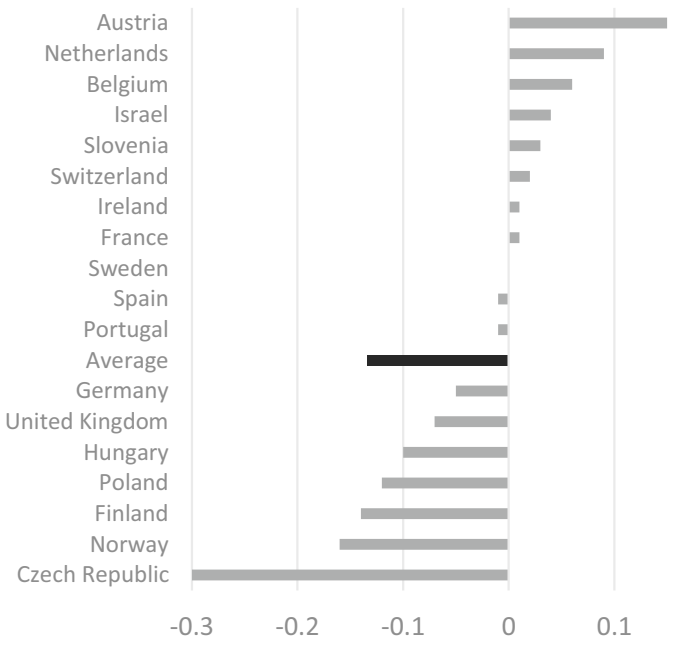


Fig. 1 Average change in gender differences in values from 2002/04 to 2014/16 for 17 European countries. The number graphed denotes the change in absolute gender difference between 2002/2004 and 2014/2016. Negative numbers mean that the difference in values have been decreasing (converging) for a specific value in a specific country whereas positive numbers indicate an increase in gender difference (divergence)

gender differences in values. In sum, we find no support for gender equality causing gender divergence/convergence in value priorities, thus refuting Hypothesis 3 (i.e., both Hypotheses $3 a$ and $3 b)$.

\section{Robustness Checks}

We also performed a series of robustness checks (with all results available in the online supplement; see Tables $2 \mathrm{~s}$ and $3 \mathrm{~s})$. To ensure that the presented results were not circumstantial, we re-ran the models using alternative measures of gender equality. First, we tested the Gender Development Index (GDI), which resulted in no significant within-country effects. Second, we tested for the possibility that an historical development of gender equality is more important than recent changes in gender equality. We tested the effect of female proportion of seats in parliament from 1960 to 2000 for the 16 countries with available data from 1960 (Paxton et al. 2008). We calculated the difference in the proportion of female-to-male seats in parliament between the years 1960 and 2000 and assigned each country with this fixed value. We then ran growth models (similar to Table 5).

Results were analogous with the GEI results reported here in that there were some between-country effects. The larger the positive difference of the female proportion of seats in parliament between 1960 and 2000, the larger the gender difference in values. There was one significant, albeit minor, interaction with time. For Power, the direct effect of seats in parliament was $b=.011(p<.001)$, with an interaction effect of $b=-.001(p=.02)$. This pattern means that countries with a larger increase in female seats in parliament from 1960 to 2000 were more likely to witness convergence in Power between men and women. There were no significant effects for Achievement, Benevolence, Stimulation, or Universalism.

Third, some previous studies have proposed that the gender gap in personality and preferences is contingent on the availability of resources (Falk and Hermle 2018; Schwartz and Rubel-Lifschitz 2009). We opted to test for economic development regardless of relative distribution between genders. We used Gross Domestic Product per capita as a proxy for economic development. Data were adopted from the World Bank (2018) and measured GDP in current USD (2018) per capita. There were no within-country effects, that is, values were unaffected by changes in GDP. (Results are available in Table $4 \mathrm{~s}$ in our online supplement.)

Finally, we examined convergence/divergence in relation to gender equality by using three waves of the International Social Survey Programme (ISSP 1999, 2013, 2017) for 46 countries $(1997,2005,2015)$. (Methods and results are available in our online supplement.) ISSP data have two advantages over ESS: it allows for a slightly longer time-period as well as the inclusion of more countries, including countries outside Europe. The disadvantages are that ISSP data have fewer time points and do not include any measures of basic values or personality traits. The latter was resolved by using ISSP-items that capture altruistic work values. We regard this as a proxy for benevolence in the work domain because the index shows high internal reliability and displays expected gender differences in almost all countries (with women attributing a stronger weight to altruistic work values than men). The analysis showed no significant effect of gender equality on within-country change in altruistic work values (see Table $6 \mathrm{~s}$ in our online supplement). In sum, robustness checks were in line with our main analyses, providing no evidence that gender equality causes any divergence or convergence in

Table 3 Repeated measurement models for each value (absolute gender differences), time only

\begin{tabular}{|c|c|c|c|c|c|c|}
\hline & Benevolence & Universalism & Achievement & Power & Stimulation & Total difference \\
\hline Model & $b(S E)$ & $b(S E)$ & $b(S E)$ & $b(S E)$ & $b(S E)$ & $b(S E)$ \\
\hline Intercept & $.21 * *(.01)$ & $.15 * *(.01)$ & $.22 * *(.02)$ & $.23 * *(.02)$ & $.22 * *(.02)$ & $1.04 * *(.05)$ \\
\hline Time & $-.0036 *(.002)$ & $-.0012(.001)$ & $-.0057 * *(.002)$ & $-.0055^{* *}(.002)$ & $-.0029(.003)$ & $-.019 * *(.006)$ \\
\hline \multicolumn{7}{|l|}{ Repeated: } \\
\hline Variance & $.0018 * *(.0002)$ & $.0013 * *(.0002)$ & $.0029 * *(.0004)$ & $.0021 * *(.0003)$ & $.0035(.0004)$ & $.023 * *(.003)$ \\
\hline Intercept & $.004 * *(.002)$ & $.0033 * *(.003)$ & $.0047 * *(.002)$ & $.011 * *(.0004)$ & $.0048(.003)$ & $.071 * *(.02)$ \\
\hline Covariance & $-.0001(.0002)$ & $-.00007(.0001)$ & $.00002(.0002)$ & $-.0008 * *(.0004)$ & $-.0004(.0003)$ & $-.0021(.002)$ \\
\hline Slope & $.00009(.00002)$ & $.00001(.00005)$ & $.00008(.00003)$ & $.0001 *(.00005)$ & $.00007(.00006)$ & $.0002(.0003)$ \\
\hline
\end{tabular}

Note: The upper part of the table shows the relationship between time and values expressed as $b$ coefficients. For example, Time $b=-.0036$ for Benevolence means that gender differences are decreasing (converging) with .0036 units per time point ( 2 years). The lower part of the table displays the variance for the repeated measurement as well as the variance for intercept, slope and the covariance between intercept and slope

$* p<.05 . * * p<.01$ 
Table 4 Repeated measurement models for each value type (absolute gender differences)

\begin{tabular}{|c|c|c|c|c|c|c|}
\hline & Benevolence & Universalism & Achievement & Power $^{\mathrm{a}}$ & Stimulation & Total $^{\mathrm{a}}$ Difference \\
\hline Model & $b(S E)$ & $b(S E)$ & $b(S E)$ & $b(S E)$ & $b(S E)$ & $b(S E)$ \\
\hline Intercept & $-.12 *(.06)$ & $-.06(.07)$ & $.06(.01)$ & $-.12(.11)$ & $.13(.08)$ & $-.16(.34)$ \\
\hline Time & $-.002(.004)$ & $-.002(.004)$ & $-.003(.006)$ & $-.01 *(.004)$ & $.009(.005)$ & $-.01(.01)$ \\
\hline GEI (between) & $.39 * *(.07)$ & $.26 * *(.08)$ & $.18(.11)$ & $.45 * *(.14)$ & $.03(.09)$ & $1.38 * *(.4)$ \\
\hline GEI (within) & $-.24(.33)$ & $.16(.30)$ & $-.34(.46)$ & $.34(.36)$ & $-1.16^{*}(.46)$ & $-1.05(.9)$ \\
\hline \multicolumn{7}{|l|}{ Repeated } \\
\hline Diagonal & $.003 * *(.0004)$ & $.002 * *(.0004)$ & $.005 * *(.001)$ & $.003 * *(.0005)$ & $.005 * *(.001)$ & $.024 * *(.003)$ \\
\hline Rho & $-.24(.16)$ & $.33 * *(.014)$ & $.48 * *(.12)$ & $.25^{*}(.1)$ & $.47 * *(.1)$ & $-.006(.11)$ \\
\hline Intercept + time & $.00003(.00002)$ & $.00007 *(.00003)$ & $.0001(.00006)$ & $.004 * *(.004)$ & $.00002(.00003)$ & $.04 * *(.01)$ \\
\hline
\end{tabular}

Note. GEI = Gender Equality Index. The upper part of the table shows the relationships among time, gender equality, and values expressed as $b$ coefficients. For example, GEI between $b=.39$ for Benevolence means that gender differences are on average .39 larger in countries with a full unit difference in GEI. GEI within for Stimulation $(b=-1.16)$ means that an increase of one unit in GEI decreases the gender difference in Stimulation with, on average, 1.16 units. The lower part of the table displays the variance part for the repeated measurement (Diagonal and Rho) as well as the variance for the intercept and time

${ }^{a}$ Power and total difference was run without a random slope for time due to model conversion issues

$* p<.05 . * * p<.01$

values; that is, we again found no support for Hypotheses $3 \mathrm{a}$ or $3 b$.

\section{Discussion}

The GEPP is the correlative finding that countries with higher gender equality display larger gender differences in personality. In the present article, we used cross-sectional time series data for 32 countries and analyzed the effect of gender equality on gender differences in basic values between 2002 and 2016. We replicated previous cross-sectional studies (e.g.,
Schwartz and Rubel-Lifschitz 2009) in finding a positive time-invariant correlation between country-level gender equality and gender differences in value priorities.

Looking across time, we found evidence of a gender convergence rather than divergence in values, with an average $15 \%$ reduction in Cohen's $d$ between first and last measurement for the five value domains. Our longitudinal analysis showed that the convergence was most palpable for the values Power, Benevolence, and Achievement, whereas the gender gap in Universalism and Stimulation remained at the same relative size.

Most important, the process of convergence reported previously was not related to prior changes in, nor onset levels of,

Table 5 Repeated measurement models for each value type (absolute gender differences), growth rates

\begin{tabular}{|c|c|c|c|c|c|}
\hline & Benevolence & Universalism & Achievement & Power $^{\mathrm{a}}$ & Stimulation \\
\hline Models & $b(S E)$ & $b(S E)$ & $b(S E)$ & $b(S E)$ & $b(S E)$ \\
\hline Intercept & $-.016(.09)$ & $-.12(.09)$ & $.25(.14)$ & $-.16(.13)$ & $.30 *(.14)$ \\
\hline Time & $-.021(.02)$ & $.027(.02)$ & $-.067(.03)$ & $-.007(.02)$ & $-.021(.03)$ \\
\hline GEI 2002 & $.29 *(.1)$ & $.34 * *(.1)$ & $-.019(.16)$ & $.49 * *(.16)$ & $-.11(.17)$ \\
\hline GEI $x$ time & $.019(.03)$ & $-.033(.03)$ & $.072(.04)$ & $-.016(.03)$ & $.022(.04)$ \\
\hline \multicolumn{6}{|l|}{ Repeated: } \\
\hline Diagonal & $.003 * *(.0004)$ & $.002 * *(.0004)$ & $.005 * *(.001)$ & $.003 * *(.0005)$ & $.006 * *(.001)$ \\
\hline Rho & $.20(.15)$ & $.33 *(.13)$ & $.45 * *(.12)$ & $.27 *(.1)$ & $.47 * *(.11)$ \\
\hline Intercept + time & $.00003 *(.00002)$ & $.00007 *(.00003)$ & $.0001 *(.00005)$ & $.004 * *(.001)$ & $.00003(.00003)$ \\
\hline
\end{tabular}

Note. GEI $=$ Gender Equality Index. The upper part of the table shows $b$ coefficients for each human value on time, gender equality in 2002, and the interaction effect between time and the GEI 2002. For example, GEI $2002 b=.29$ for Benevolence means that gender differences are on average .29 larger in countries with a full unit difference in GEI 2002. The GEI x Time interaction shows the $b$ coefficients for the interaction between GEI and time. The lower part of the table displays the variance part for the repeated measurement (Diagonal and Rho) as well as the variance for the intercept and time

${ }^{a}$ Power was run without a random slope for time due to model conversion issues

$* p<.05 . * * p<.01$ 
gender equality. Even though the period also saw a general increase in country levels of gender equality, we found no evidence of a systematic relationship between progress in gender equality and change in values. Thus, neither of the competing predictions from evolutionary and biosocial perspectives were supported in this regard because we cannot state that gender equality drives gender differences in values.

Our results indicate that the correlational observation underlying the GEPP represents a spurious relationship between gender equality and differences in personality across countries. One possibility is that the observed correlative pattern is due to chance. However, given the stability of the crosssectional findings, considering especially the multitude of measures of gender equality and personality as well as evidence from various geographical settings, this is unlikely. It is more likely that there exist confounding factors that relate both to gender equality and personality development. We believe this conclusion is the most serious contribution of our findings, and consequently we encourage future research to focus on such aspects. For example, a recent study by Kaiser (2019) indicates that cultural individualism, food consumption, and historical levels of pathogen prevalence may be such confounding factors.

\section{Limitations and Directions for Future Research}

We acknowledge several limitations to the results presented in the present paper. First, the time horizon of our analysis was relatively short and recent. However, we still observed meaningful trends in values as well as variation in gender equality during this period. The fact that we do observe convergence in value priorities speaks to the fact that our time span, although short, was enough to capture changes to gender differences in values as one aspect of personality. Another limitation is the possibility that the time lag from changes in gender equality to changes in gender differences in personality is longer than the 14 -year period we analyzed. Although our analysis using historical data does not support this idea, our measure was not exhaustive.

Second, we need to consider our operationalization of gender equality. This definition is most important to the biosocial theoretical perspective, which holds that gender differences continue to linger due to progression in gender equality in formal institutions being unmatched by a similar progression in the division of unpaid labor and family obligations (Wood and Eagly 2012). Unfortunately, although widely accepted as a proper measure of gender equality, the Gender Inequality Index does not include measures of equality of time allocation or unpaid care and domestic work. There is, unfortunately, serious limitations to the existence of measures for all or most of the studied countries across several measurement points. However, within Europe it has been observed that (in)equality in the division of unpaid labor generally follows the same pattern as other indicators of gender equality (European Institute for Gender Equality 2017). Thus, we argue it likely that inclusions of such measures, were they readily available, would not alter conclusions based on our findings.

Third, our operationalization of personality as value priorities requires examination. Although not equivalent to traits, we argue that values are an integral part of a person's psychological makeup. Values also provide a good middle ground between previously studied higher-order traits and lowerorder aspects of personality such as preferences. In addition, measurement indicators overlap to a considerable extent, as do the correlations between traits and value domains (Fischer and Boer 2015). Moreover, even when treated as entirely separate, the fact remains that the observed cross-sectional relationship between gender equality and gender differences in value priorities closely mimic that of both traits and preferences (Schwartz and Rubel-Lifschitz 2009). The present study corroborates this conclusion because our findings were similar when focusing on work values in addition to basic value priorities.

In summation, we argue that future research should focus on identifying alternative factors, other than gender equality, that drive a change in gender differences in personality. Ideally, such research should rely on a longer time series for personality and more countries as well as utilize historical data for independent variables.

\section{Practice Implications}

Researchers from different fields claim that progression in gender equality alters the socio-structural environment, which has bearing on personality development, and ultimately affects observed gender differences in personality, either through suppressing or aiding innate differences or by actually shaping personality through internalization. We have demonstrated that such claims, and any policy or practice implications based on them, must be taken with a great deal of caution as there is no observable link across time between changes in gender equality and gender differences in personality. At the very least, such changes are very slow to materialize. For instance, our findings make it uncertain whether the gender gap in STEM and EHW educations and occupations can be attributed to increased gender differences in personality following a progression in gender equality. Therefore, policy professionals within labor markets and education cannot act on gender differentiated outcomes in these areas as something predetermined by general human development.

\section{Conclusion}

The Gender-Equality-Personality Paradox has received much scholarly attention. It has also been prominent in the wider 
public debate on gender differences. However, previous studies are limited because the approach has been to study this relationship either cross-sectionally or over time using only one or a few countries. The present findings, using a longitudinal approach, poses serious questions regarding previous studies' causal framing of the relationship between gender equality and gender differences in personality. We do find evidence both of a positive correlation between countries' level of gender equality and gender differences in values, while also finding that, for most countries, value priorities converge over time. However, the lack of any systematic development in the relationship between gender equality and gender differences in values means that it is highly questionable whether gender equality really drives observed changes in gender differences in values (as one aspect of personality). Thus, we argue that it is time to rethink the relationship between gender equality and gender differences in personality by exploring alternative explanations.

Acknowledgements Open access funding provided by Umea University.

\section{Compliance with Ethical Standards}

Data used in our article involved human subjects who consented to participate in the ESS and ISSP-studies.

Our article reflect original work not published or submitted elsewhere.

Conflict of Interest There are no conflicts of interest.

Open Access This article is distributed under the terms of the Creative Commons Attribution 4.0 International License (http:// creativecommons.org/licenses/by/4.0/), which permits unrestricted use, distribution, and reproduction in any medium, provided you give appropriate credit to the original author(s) and the source, provide a link to the Creative Commons license, and indicate if changes were made.

\section{References}

Bjorklund, D. F., \& Shackelford, T. K. (1999). Differences in parental investment contribute to important differences between men and women. Current Directions in Psychological Science, 8(3), 86-89. https://doi.org/10.1111/1467-8721.00020.

Boxer, C. F., Noonan, M., \& Whelan, C. B. (2013). Measuring mate preferences: A replication and extension. Journal of Family Issues, 36(2), 163-187. https://doi.org/10.1177/0192513X13490404.

Donnelly, K., \& Twenge, J. M. (2017). Masculine and feminine traits on the Bem Sex-Role Inventory, 1993-2012: A cross-temporal metaanalysis. Sex Roles, 76(9-10), 556-565. https://doi.org/10.1007/ s11199-016-0625-y.

Eagly, A. H., \& Karau, S. J. (1991). Gender and the emergence of leaders: A meta-analysis. Journal of Personality and Social Psychology, 60(5), 685-710. https://doi.org/10.1037/0022-3514.60.5.685.

Eagly, A. H., Wood, W., \& Diekman, A. B. (2000). Social role theory of sex differences and similarities: A current appraisal. In T. Eckes \& H. M. Trautner (Eds.), The developmental social psychology of gender (pp. 123-174). Mahwah: Erlbaum.
European Institute for Gender Equality. (2017). Gender equality index, 2017. Retrieved from https://eige.europa.eu/gender-equality-index.

European Social Survey Cumulative File, ESS 1-8. (2016). Data file edition 1.0. NSD - Norwegian Centre for Research Data, Norway - Data Archive and distributor of ESS data for ESS ERIC. https:// doi.org/10.21338/NSD-ESS-CUMULATIVE.

Falk, A., \& Hermle, J. (2018). Relationship of gender differences in preferences to economic development and gender equality. Science, 362, 1-6. https://doi.org/10.1126/science.aas9899.

Fischer, R., \& Boer, D. (2015). Motivational basis of personality traits: A meta-analysis of value-personality correlations. Journal of Personality, 83(5), 491-510. https://doi.org/10.1111/jopy.12125.

Grijalva, E., Newman, D. A., Tay, L., Donnellan, M. B., Harms, P. D., Robins, R. W., ... Yan, T. (2015). Gender differences in narcissism: A meta-analytic review. Psychological Bulletin, 141(2), 261-310. https://doi.org/10.1037/a0038231.

Hopcroft, R. L., \& Bradley, D. B. (2007). The sex difference in depression across 29 countries. Social Forces, 85(4), 1484-1507. https:// doi.org/10.1353/sof.2007.0071.

ISSP Research Group. (1999). International social survey programme: Work orientations II - ISSP 1997. GESIS Data Archive, Cologne. ZA3090 Data file Version 1.0.0. https://doi.org/10.4232/1.3090.

ISSP Research Group. (2013). International social survey programme: Work orientation III - ISSP 2005. GESIS Data Archive, Cologne. ZA4350 Data file Version 2.0.0. https://doi.org/10.4232/1.11648.

ISSP Research Group. (2017). International social survey programme: Work orientations IV - ISSP 2015. GESIS Data Archive, Cologne. ZA6770 Data file Version 2.1.0. https://doi.org/10.4232/1.12848.

Kaiser, T. (2019). Nature and evoked culture: Sex differences in personality are uniquely correlated with ecological stress. Personality and Individual Differences, 148, 67-72. https://doi.org/10.1016/j.paid. 2019.05.011.

Kajonius, P. J., \& Johnson, J. (2018). Sex differences in 30 facets of the five factor model of personality in the large public $(\mathrm{N}=320,128)$. Personality and Individual Differences, 129, 126-130. https://doi. org/10.1016/j.paid.2018.03.026.

Koch, A. (2018). Assessment of socio-demographic sample composition in ESS Round 7, Working paper. GESIS: Mannheim Retrieved from https://www.europeansocialsurvey.org/docs/round7/methods/ ESS7_sample_composition_assessment.pdf.

Konrad, A. M., Ritchie Jr., J. E., Lieb, P., \& Corrigal, E. (2000). Sex differences and similarities in job attribute preferences: A meta-analysis. Psychological Bulletin, 126(4), 593-641. https://doi.org/10. 1037/0033-2909.126.4.593.

Mac Giolla, E., \& Kajonius, P. J. (2018). Sex differences in personality are larger in gender equal countries: Replicating and extending a surprising finding. International Journal of Psychology. Advance online publication. https://doi.org/10.1002/ijop.12529.

Paxton, P., Green, J., \& Hughes, M. M. (2008). Women in parliament, 1945-2003: Cross-national dataset. Ann Arbor: Inter-university Consortium for Political and Social Research. https://doi.org/10. 3886/ICPSR24340.v1.

Piurko, Y., Schwartz, S. H., \& Davidov, E. (2011). Basic personal values and the meaning of left-right political orientations in 20 countries. Political Psychology, 32(4), 537-561. https://doi.org/10.1111/j. 1467-9221.2011.00828.x.

Schmitt, D. P., Realo, A., Voracek, M., \& Alik, J. (2008). Why can't a man be more like a woman? Sex differences in Big Five personality traits across 55 cultures. Journal of Personality and Social Psychology, 94, 168-192. https://doi.org/10.1037/a0014651.

Schmitt, D. P., Alcalay, L., Allik, J., Alves, I. C. B., Anderson, C. A., Angelini, A. L., ... Youn, G. (2016). Psychological sex differences across cultures: Findings from the International Sexuality Description Project-2. Manuscript in preparation.

Schmitt, D. P., Long, A. E., Mcphearson, A., O'Brien, K., Remmert, B., \& Shah, S. H. (2017). Personality and gender differences in global 
perspective. International Journal of Psychology, 51(1), 45-56. https://doi.org/10.1002/ijop.12265.

Schwartz, S. H. (1992). Universals in the content and structure of values: Theory and empirical tests in 20 countries. In M. Zanna (Ed.), Advances in experimental social psychology (Vol. 25, pp. 1-65). New York: Academic.

Schwartz, S. (2003). A proposal for measuring value orientations across nations. Questionnaire Package of ESS, 259-290 Retrieved from https://www.europeansocialsurvey.org/docs/methodology/core ess questionnaire/ESS core questionnaire human values.pdf.

Schwartz, S. H. (2007). Value orientations: Measurement, antecedents and consequences across nations. In J. Jowell, C. Roberts, R. Fitzgerald, \& G. Eva (Eds.), Measuring attitudes cross-nationally: Lessons from the European Social Survey (pp. 161-193). Los Angeles: Sage. https://doi.org/10.4135/9781849209458.n9.

Schwartz, S. H. (2012). An overview of the Schwartz theory of basic values. Online Readings in Psychology and Culture, 2(1), 1-20. https://doi.org/10.9707/2307-0919.1116.

Schwartz, S. H., \& Rubel, T. (2005). Sex differences in value priorities: Cross-cultural and multimethod studies. Journal of Personality and Social Psychology, 89(6), 1010-1028. https://doi.org/10.1037/ 0022-3514.89.6.1010.

Schwartz, S. H., \& Rubel-Lifschitz, T. (2009). Cross-national variation in the size of sex differences in values: Effects of gender equality. Journal of Personality and Social Psychology, 97, 171-187. https://doi.org/10.1037/a0015546.

Stoet, G., \& Geary, D. C. (2018). The gender-equality paradox in science, technology, engineering, and mathematics education. Psychological Science, 29(4), 581-593. https://doi.org/10.1177/ 0956797617741719.

The World Bank Group. (2018). GDP current US\$. World Bank national accounts data, and OECD National Accounts data files. Retrieved from https://data.worldbank.org/indicator/NY.GDP.MKTP.CD.

Twenge, J. M. (1997). Changes in masculine and feminine traits over time: A meta-analysis. Sex Roles, 36, 305-325. https://doi.org/10. 1007/BF02766650.

Twenge, J. M., \& Campbell, W. K. (2002). Self-esteem and socioeconomic status: A meta-analytic review. Personality and Social Psychology Review, 6, 59-71. https://doi.org/10.1207/ S15327957PSPR0601_3.
Twenge, J. M., Konrath, S., Foster, J. D., Campbell, W. K., \& Bushman, B. J. (2008). Egos inflating over time: A cross-temporal meta-analysis of the Narcissistic Personality Inventory. Journal of Personality, 76, 875-902. https://doi.org/10.1111/j.1467-6494.2008.00507.x.

U.S. Census Bureau. (2012). The 2012 statistical abstract: The national data book. Retrieved from http://www.census.gov/prod/2011pubs/ 12statab/labor.pdf.

United Nations Development Programme. (2018). Human development indices and indicators: 2018 statistical update. Table 5: Gender inequality index. Retrieved from http://hdr.undp.org/en/content/ gender-inequality-index-gii.

Uzefovsky, F., Döring, A. K., \& Knafo-Noam, A. (2016). Values in middle childhood: Social and genetic contributions. Social Development Quartet, 25(3), 482-502. https://doi.org/10.1111/sode.12155.

Wang, M. T., \& Degol, J. L. (2017). Gender gap in science, technology, engineering, and mathematics (STEM): Current knowledge, implications for practice, policy, and future directions. Educational Psychology Review, 29, 119-140. https://doi.org/10.1007/s10648015-9355-x.

Wood, W., \& Eagly, A. H. (2012). Biosocial construction of sex differences and similarities in behaviour. In J. M. Olson \& M. P. Zanna (Eds.), Advances in experimental social psychology (Vol. 46, pp. 55-123). Burlington: Academic.

Zell, E., Krizan, Z., \& Teeters, S. R. (2015). Evaluating gender similarities and differences using metasynthesis. American Psychologist, 70(1), 10-20. https://doi.org/10.1037/a0038208.

Zuckerman, M., Li, C., \& Hall, J. A. (2016). When men and women differ in self-esteem and when they don't: A meta-analysis. Journal of Research in Personality, 64, 34-51. https://doi.org/10.1016/j.jrp. 2016.07.007.

Zuckerman, M., Li, C., \& Diener, E. F. (2017). Societal conditions and the gender difference in well-being: Testing a three-stage model. Personality and Social Psychological Bulletin, 43(3), 329-336. https://doi.org/10.1177/0146167216684133.

Publisher's Note Springer Nature remains neutral with regard to jurisdictional claims in published maps and institutional affiliations. 